\title{
Stochastic Averaging for Stochastic Differential Equations Driven by Fractional Brownian Motion and Standard Brownian Motion
}

\author{
Bin $\mathrm{Pei}^{\mathrm{a}, \mathrm{b}}$, Yong $\mathrm{Xu}^{\mathrm{c}, *}$, Jiang-Lun $\mathrm{Wu}^{\mathrm{d}}$ \\ ${ }^{a}$ School of Mathematical Sciences, Fudan University, Shanghai, 200433, China \\ ${ }^{b}$ Graduate School of Mathematics, Kyushu University, Fukuoka, 819-0395, Japan \\ ${ }^{c}$ Department of Applied Mathematics, Northwestern Polytechnical University, Xi'an, 710072, China \\ ${ }^{d}$ Department of Mathematics, Swansea University, Swansea, SA1 8EN, UK
}

\begin{abstract}
In this paper, an averaging principle for multidimensional, time dependent, stochastic differential equations (SDEs) driven by fractional Brownian motion and standard Brownian motion was established. We combined the pathwise approach with the Itô stochastic calculus to handle both types of integrals involved and proved that the original SDEs can be approximated by averaged SDEs in the manner of mean square convergence and of convergence in probability, respectively.
\end{abstract}

Keywords: Averaging principle, fractional Brownian motion, pathwise Riemann-Stieltjes integral, Itô stochastic calculus.

2010 MSC: 60G22, 60H10

\section{Introduction}

The fractional Brownian motion ( $\mathrm{fBm}$ ) with Hurst index $H \in(0,1)$ is a zero mean Gaussian process $\left\{B_{t}^{H}, t \geq 0\right\}$ with covariance function $R_{H}(s, t)=\frac{1}{2}\left(s^{2 H}+t^{2 H}-|t-s|^{2 H}\right), s, t \in(0, \infty)$. This process was introduced by Kolmogorov [1] and later studied by Mandelbrot and Van Ness [2]. Its self-similarity and long-range dependence $H>\frac{1}{2}$ properties make this process a very useful driving noise in modelings arising in physics, finance and many other fields [10].

The present paper focuses on the following stochastic differential equations (SDEs) driven by fBm and standard Brownian motion $(\mathrm{Bm})$ on $\mathbb{R}^{d}$ :

$$
X_{t}=X_{0}+\int_{0}^{t} f\left(s, X_{s}\right) d s+\int_{0}^{t} \sigma_{W}\left(s, X_{s}\right) d W_{s}+\int_{0}^{t} \sigma_{H}\left(s, X_{s}\right) d B_{s}^{H},
$$

where $X_{0}$ is a $d$-dimensional random variable independent of $W$ and $B^{H}$ with $\mathbb{E}\left|X_{0}\right|^{2}<\infty, B^{H}=\left\{B_{t}^{H}, t \in\right.$ $[0, \infty)\}$ is an $m$-dimensional $\mathrm{fBm}$ with Hurst parameter $H \in\left(\frac{1}{2}, 1\right)$, and $W=\left\{W_{t}, t \in[0, \infty)\right\}$ is an $r$ dimensional standard Bm, independent of $B^{H}$. The integral $\int \cdot d W$ should be interpreted as an Itô stochastic integral, and the integral $\int \cdot d B^{H}$ as a pathwise Riemann-Stieltjes integral in the sense of Zähle $[3,4,5]$. The coefficients are jointly measurable functions $f^{i}, \sigma_{W}^{i, k}, \sigma_{H}^{i, j}:[0, \infty) \times \mathbb{R}^{d} \rightarrow \mathbb{R}, 1 \leq i \leq d, 1 \leq k \leq r, 1 \leq j \leq m$. For arbitrarily fixed $T>0$, we will make use of the following assumptions on the coefficients of Eq.(1.1).

- (H1) The function $\sigma_{H}(t, x)$ is continuously differentiable in the variable $x \in \mathbb{R}^{d}$, for each $t \in[0, T]$. Moreover, there exist constants $L_{i}, i=1,2,3,4$, such that $\left|\sigma_{H}(t, x)-\sigma_{H}(t, y)\right| \leq L_{1}|x-y|, \mid \partial_{x_{i}} \sigma_{H}(t, x)-$ $\partial_{x_{i}} \sigma_{H}(t, y)\left|\leq L_{2}\right| x-\left.y\right|^{\delta},\left|\partial_{x_{i}} \sigma_{H}(t, x)-\partial_{x_{i}} \sigma_{H}(s, x)\right|+\left|\sigma_{H}(t, x)-\sigma_{H}(s, x)\right| \leq L_{3}|t-s|^{\beta},\left|\partial_{x_{i}} \sigma_{H}(t, x)\right| \leq$ $L_{4}$, for all $x, y \in \mathbb{R}^{d}, t \in[0, T]$, and for some constants $0 \leq \beta, \delta \leq 1$.

- (H2) The functions $f(t, x)$ and $\sigma_{W}(t, x)$ are Lipschitz continuous in the variable $x$ and have linear growth in the same variable, uniformly in $t \in[0, T]$. Moreover, there exist constants $L_{i}, i=5,6$, such that $\left.|f(t, x)-f(t, y)|+\left|\sigma_{W}(t, x)-\sigma_{W}(t, y)\right| \leq L_{5}|x-y|, \mid f(t, x)\right)|+| \sigma_{W}(t, x) \mid \leq L_{6}(1+|x|)$.

\footnotetext{
${ }^{*}$ Corresponding author

Email addresses: binpei@hotmail.com (Bin Pei), hsux3@nwpu.edu.cn (Yong Xu ), j.l.wu@swansea.ac.uk (Jiang-Lun Wu)
} 
Note that Assumption (H1) implies the linear growth property, i.e., there exists a constant $L_{7}$ such that $\left|\sigma_{H}(t, x)\right| \leq L_{7}(1+|x|)$.

Lyons [6] solved the equations driven only by a fBm with Hurst parameter $H>\frac{1}{2}$ by a pathwise approach using the $p$-variation norm in the framework of rough path theory. Nualart and Răşcanu [4] studied the differential equations driven by fBm using the tools of fractional calculus in the sense of Zähle [3]. Kubilius [7] studied one dimensional SDEs driven by both $\mathrm{fBm}$ and standard $\mathrm{Bm}$, with $\sigma_{W}, \sigma_{H}$ independent of the time variable and with no drift term $(f \equiv 0)$. Guerra and Nualart [5] established an existence and uniqueness theorem for solutions of multidimensional, time dependent, SDEs driven by fBm with Hurst parameter $H>\frac{1}{2}$ and standard Bm.

Stochastic averaging, which is usually used to approximate dynamical systems under random fluctuations, has a long and rich history in multiscale problems, see e.g. $[11,14,16,17,12,13]$ and references therein. Xu et al. $[8,15]$ developed a stochastic averaging technique for SDEs with $\mathrm{fBm}\left(\sigma_{W}=0\right.$ in Eq.(1.1)) and proved that the original SDEs can be approximated by averaged SDEs in the sense of mean square convergence and of convergence in probability. The main difficulties here are how to deal with both $\mathrm{fBm}$ and standard $\mathrm{Bm}$. In order to overcome these difficulties, our approach is completely different from Xu's previous work $[8,15]$ in the sense that we combine the pathwise approach with the Itô stochastic calculus to handle both types of integrals and we established an averaging principle for multidimensional, time dependent, SDEs (1.1) with fBm $H>\frac{1}{2}$ and standard Bm.

The rest of the paper is arranged as follows. Section 2 presents preliminary results that are needed in the subsequent section. In Section 3, we obtained stochastic averaging for SDEs driven by fBm $\left(H>\frac{1}{2}\right)$ and standard Bm.

\section{Preliminaries}

Let $(\Omega, \mathcal{F}, \mathbb{P})$ be a complete probability space . For each $t \in[0, T]$, we denote by $\mathcal{F}_{t}$ the $\sigma$-field generated by the random variables $\left\{X_{0}, B_{s}^{H}, W_{s}, s \in[0, t]\right\}$ and all $P$-null sets. In addition to the natural filtration $\mathcal{F}_{t}, t \in[0, T]$, we will consider a larger filtration $\mathcal{G}_{t}, t \in[0, T]$ such that $\left\{\mathcal{G}_{t}\right\}$ is right-continuous and $\left\{\mathcal{G}_{0}\right\}$ contains the $P$-null sets, so that $X_{0}, B^{H}$ are $\mathcal{G}_{0}$-measurable, and $W$ is a $\mathcal{G}_{t}$-Brownian motion. Notice that $\hat{\mathcal{F}}_{t} \subset \mathcal{G}_{t}$, where $\hat{\mathcal{F}}_{t}$ is the $\sigma$-field generated by the random variable $\left\{X_{0}, B^{H}, W_{s}, s \in[0, t]\right\}$ and the $P$-null sets.

For $H \in\left(\frac{1}{2}, 1\right)$, let $1-H<\alpha<\frac{1}{2}$, then, denote by $W_{0}^{\alpha, \infty}$ the space of measurable functions $f(t)$ : $[0, T] \rightarrow \mathbb{R}^{d}$ such that $\|f\|_{\alpha, \infty}:=\sup _{t \in[0, T]}\|f(t)\|_{\alpha}<\infty$, where

$$
\|f(t)\|_{\alpha}:=|f(t)|+\int_{0}^{t} \frac{|f(t)-f(s)|}{(t-s)^{\alpha+1}} d s .
$$

For $\mu \in(0,1]$, let $C^{\mu}$ be the space of $\mu$-Hölder continuous functions $f:[0, T] \rightarrow \mathbb{R}^{d}$, equipped with the the norm

$$
\|f\|_{\mu}:=\|f\|_{\infty}+\sup _{0 \leq s<t \leq T} \frac{|f(t)-f(s)|}{(t-s)^{\mu}},\|f\|_{\infty}=\sup _{0 \leq t \leq T}|f(t)| .
$$

Given any $\epsilon$ such that $0<\epsilon<\alpha$, we have the following inclusions $C^{\alpha+\epsilon} \subset W_{0}^{\alpha, \infty} \subset C^{\alpha-\epsilon}$. Now, fix the parameter $\alpha$ such that $0<\alpha<\frac{1}{2}$, denote by $W_{T}^{1-\alpha, \infty}$ the space of measurable functions $g(t):[0, T] \rightarrow \mathbb{R}^{m}$ such that

$$
\|g\|_{1-\alpha, \infty, T}:=\sup _{0<s<t<T}\left(\frac{|g(t)-g(s)|}{(t-s)^{1-\alpha}}+\int_{s}^{t} \frac{|g(y)-g(s)|}{(y-s)^{2-\alpha}} d y\right)<\infty .
$$

And denote by $W_{0}^{\alpha, 1}$ the space of measurable functions $f(t):[0, T] \rightarrow \mathbb{R}^{d}$ such that

$$
\|f\|_{\alpha, 1}:=\int_{0}^{T} \frac{|f(s)|}{s^{\alpha}} d s+\int_{0}^{T} \int_{0}^{s} \frac{|f(s)-f(y)|}{(s-y)^{\alpha+1}} d y d s<\infty .
$$

It is easy to prove that $C^{1-\alpha+\epsilon} \subset W_{T}^{1-\alpha, \infty} \subset C^{1-\alpha}$. For $g \in W_{T}^{1-\alpha, \infty}$, we have that

$$
\Lambda_{\alpha}(g):=\frac{1}{\Gamma(1-\alpha)} \sup _{0<s<t<T}\left|\left(D_{t-}^{1-\alpha} g_{t-}\right)(s)\right| \leq \frac{1}{\Gamma(1-\alpha) \Gamma(\alpha)}\|g\|_{1-\alpha, \infty, T}<\infty,
$$

where $\Gamma(\cdot)$ is the Gamma function and $D_{t-}^{1-\alpha}$ stands for the Weyl derivative $[4,17]$. Moreover, if $f \in W_{0}^{\alpha, 1}$ and $g \in W_{T}^{1-\alpha, \infty}$ then $\int_{0}^{t} f d g$ exists for all $t \in[0, T]$ and $\left|\int_{0}^{t} f d g\right| \leq \Lambda_{\alpha}(g)\|f\|_{\alpha, 1}$. 
We denote by $\mathbb{E}$ the condition expectation given $\hat{\mathcal{F}}_{0}$, that is, given $X_{0}$ and $B^{H}$. We now define the space of processes where we will search for solutions of (1.1).

Remark 2.1. The trajectories of $B^{H}$ are almost surely locally $\alpha$-Hölder continuous for all $\alpha \in(0, H)$. Then, the trajectories of $B^{H}$ belong to the space $W_{T}^{1-\alpha, \infty}$. Consequently, the pathwise Riemann-Stieltjes integral $\int_{0}^{T} v_{s} d B_{s}^{H}$ exists if $\left\{v_{t}, t \in[0, T]\right\}$ is a stochastic process whose trajectories belong to the space $W_{0}^{\alpha, 1}$ with $1-H<\alpha<\frac{1}{2}$. And we have the following estimate

$$
\left|\int_{0}^{t} v_{s} d B_{s}^{H}\right| \leq \Lambda_{\alpha}\left(B^{H}\right)\|v\|_{\alpha, 1},
$$

where $\Lambda_{\alpha}\left(B^{H}\right)$ has moments of all orders, see Lemma 7.5 in Nualart and Răşcanu [4].

Definition 2.2. Let $\mathbb{W}_{\mathcal{G}}$ be the space of $d$-dimensional $\mathcal{G}_{t}$-adapted stochastic process $X=\left\{X_{t}, t \in[0, T]\right\}$ such that almost surely the trajectories of $X$ belong to $W_{0}^{\alpha, \infty}$ and $\int_{0}^{T} \mathbb{E}\left\|X_{s}\right\|_{\alpha}^{2} d s<\infty$. A strong solution of the $\operatorname{SDE}(1.1)$ is a stochastic process $X$ in the space $\mathbb{W}_{\mathcal{G}}$ which satisfies Eq. (1.1).

Next, according to Theorem 2.2 in [5], we have the following lemma.

Lemma 2.3. Suppose that Eq. (1.1) satisfies the conditions (H1)-(H2), then, for $1-H<\alpha<\min \left\{\frac{1}{2}, \beta, \frac{\delta}{2}\right\}, H \in$ $\left(\frac{1}{2}, 1\right)$, the Eq. (1.1) has a unique strong solution $X_{t}$.

\section{The Stochastic Averaging Principle}

Fix $\varepsilon_{0}>0$, we set, for each $\varepsilon \in\left(0, \varepsilon_{0}\right]$, the following standard SDE $a$ la Eq.(1.1):

$$
X_{t}^{\varepsilon}=X_{0}+\varepsilon \int_{0}^{t} f\left(s, X_{s}^{\varepsilon}\right) d s+\sqrt{\varepsilon} \int_{0}^{t} \sigma_{W}\left(s, X_{s}^{\varepsilon}\right) d W_{s}+\varepsilon^{H} \int_{0}^{t} \sigma_{H}\left(s, X_{s}^{\varepsilon}\right) d B_{s}^{H} .
$$

The coefficients of Eq (3.1) fulfill the same conditions as in (1.1). Besides, let functions $\bar{f}: \mathbb{R}^{d} \rightarrow \mathbb{R}^{d}, \bar{\sigma}_{W}$ : $\mathbb{R}^{d} \rightarrow \mathbb{R}^{d \times r}, \bar{\sigma}_{H}: \mathbb{R}^{d} \rightarrow \mathbb{R}^{d \times m}$ be continuous and they satisfy the same condition as with $f, \sigma_{W}, \sigma_{H}$, respectively. Also assume that the following additional inequalities are satisfied: (C1) $\frac{1}{T_{1}} \int_{0}^{T_{1}}|f(s, x)-\bar{f}(x)| d s \leq$ $\varphi_{1}\left(T_{1}\right)(1+|x|) ;(\mathbf{C 2}) \frac{1}{T_{1}} \int_{0}^{T_{1}}\left|\sigma_{W}(s, x)-\bar{\sigma}_{W}(x)\right|^{2} d s \leq \varphi_{2}\left(T_{1}\right)\left(1+|x|^{2}\right) ;(\mathbf{C 3}) \frac{1}{T_{1}} \int_{0}^{T_{1}}\left|\sigma_{H}(s, x)-\bar{\sigma}_{H}(x)\right|^{2} d s \leq$ $\varphi_{3}\left(T_{1}\right)\left(1+|x|^{2}\right)$, where $T_{1} \in[0, T], \varphi_{i}\left(T_{1}\right)$ are positive bounded functions with $\lim _{T_{1} \rightarrow \infty} \varphi_{i}\left(T_{1}\right)=0, i=1,2,3$.

Then, we can obtain the averaged SDEs by the averaging principle:

$$
Z_{t}^{\varepsilon}=X_{0}+\varepsilon \int_{0}^{t} \bar{f}\left(Z_{s}^{\varepsilon}\right) d s+\sqrt{\varepsilon} \int_{0}^{t} \bar{\sigma}_{W}\left(Z_{s}^{\varepsilon}\right) d W_{s}+\varepsilon^{H} \int_{0}^{t} \bar{\sigma}_{H}\left(Z_{s}^{\varepsilon}\right) d B_{s}^{H} .
$$

Under the similar conditions such as $X_{t}^{\varepsilon}$ in (3.1), this equation has a unique strong solution $Z_{t}^{\varepsilon}$.

Lemma 3.1. Suppose that the averaged Eq. (3.2) satisfies the conditions (H1)-(H2). Then, for $t \in[0, T]$, we have, $\sup _{t \in[0, T]} \mathbb{E}\left[\left\|Z_{t}^{\varepsilon}\right\|_{\alpha}^{2}\right] \leq C$.

Proof: From (3.2) and by elementary inequalities, we have

$$
\begin{aligned}
\mathbb{E}\left\|Z_{t}^{\varepsilon}\right\|_{\alpha}^{2} & \leq 4 \mathbb{E}\left|X_{0}\right|^{2}+4 \varepsilon^{2} \mathbb{E}\left\|\int_{0}^{t} \bar{f}\left(Z_{s}^{\varepsilon}\right) d s\right\|_{\alpha}^{2}+4 \varepsilon \mathbb{E}\left\|\int_{0}^{t} \bar{\sigma}_{W}\left(Z_{s}^{\varepsilon}\right) d W_{s}\right\|_{\alpha}^{2}+4 \varepsilon^{2 H} \mathbb{E}\left\|\int_{0}^{t} \bar{\sigma}_{H}\left(Z_{s}^{\varepsilon}\right) d B_{s}^{H}\right\|_{\alpha}^{2} \\
& =: 4 \mathbb{E}\left|X_{0}\right|^{2}+4 I_{t}^{1}+4 I_{t}^{2}+4 I_{t}^{3} .
\end{aligned}
$$

Firstly, by the growth conditon (H2) and Proposition 3.3 in Guerra and Nualart [5], it is not hard to obtain

$$
\begin{aligned}
I_{t}^{1} & =\varepsilon^{2} \mathbb{E}\left(\left|\int_{0}^{t} \bar{f}\left(Z_{s}^{\varepsilon}\right) d s\right|+\int_{0}^{t}\left|\int_{s}^{t} \bar{f}\left(Z_{r}^{\varepsilon}\right) d r\right|(t-s)^{-\alpha-1} d s\right)^{2} \\
& \leq C \varepsilon^{2} \mathbb{E}\left(\int_{0}^{t}\left|\bar{f}\left(Z_{s}^{\varepsilon}\right)\right| d s\right)^{2}+C \varepsilon^{2} \mathbb{E}\left(\int_{0}^{t}(t-r)^{-\alpha}\left|\bar{f}\left(Z_{r}^{\varepsilon}\right)\right| d r\right)^{2} \\
& \leq C \varepsilon_{0}^{2} \int_{0}^{t}(t-s)^{-2 \alpha} \mathbb{E}\left|Z_{s}^{\varepsilon}\right|^{2} d s+C_{\alpha, T} \varepsilon_{0}^{2} .
\end{aligned}
$$

Then, for $I_{t}^{2}$, by the growth condition (H2) and Proposition 3.8 in Guerra and Nualart [5], we have

$$
I_{t}^{2} \leq C \varepsilon \mathbb{E}\left|\int_{0}^{t} \bar{\sigma}_{W}\left(Z_{s}^{\varepsilon}\right) d W_{s}\right|^{2}+C \varepsilon \mathbb{E}\left(\int_{0}^{t} \frac{\left|\int_{s}^{t} \bar{\sigma}_{W}\left(Z_{r}^{\varepsilon}\right) d W_{r}\right|}{(t-s)^{\alpha+1}} d s\right)^{2}
$$




$$
\begin{aligned}
& \leq C \varepsilon \mathbb{E} \int_{0}^{t}\left(1+\left|Z_{s}^{\varepsilon}\right|^{2}\right) d s+C \varepsilon \mathbb{E} \int_{0}^{t}(t-s)^{-\frac{3}{2}-\alpha} \int_{s}^{t}\left|\bar{\sigma}_{W}\left(X_{r}^{\varepsilon}\right)\right|^{2} d r d s \\
& \leq C \varepsilon_{0} \int_{0}^{t}(t-s)^{-\frac{1}{2}-\alpha} \mathbb{E}\left|Z_{s}^{\varepsilon}\right|^{2} d s+C_{\alpha, T} \varepsilon_{0} .
\end{aligned}
$$

For $I_{t}^{3}$, by (2.1) and Proposition 3.5 in Guerra and Nualart [5], we have

Finally, we obtain that

$$
\begin{aligned}
I_{t}^{3} & \leq C \varepsilon^{2 H}\left(\left|\int_{0}^{t} \bar{\sigma}_{H}\left(Z_{s}^{\varepsilon}\right) d B_{s}^{H}\right|+\int_{0}^{t}\left|\int_{s}^{t} \bar{\sigma}_{H}\left(Z_{r}^{\varepsilon}\right) d B_{r}^{H}\right|(t-s)^{-\alpha-1} d s\right)^{2} \\
& \leq C \varepsilon_{0}^{2 H} \Lambda_{\alpha}\left(B^{H}\right)^{2} \int_{0}^{t}\left((t-s)^{-2 \alpha}+s^{-\alpha}\right)\left(1+\left\|Z_{s}^{\varepsilon}\right\|_{\alpha}^{2}\right) d s .
\end{aligned}
$$

$$
\begin{aligned}
\sup _{0 \leq s \leq t} \mathbb{E}\left[\left\|Z_{s}^{\varepsilon}\right\|_{\alpha}^{2}\right] & \leq C+C \int_{0}^{t}\left(\left(\frac{t}{s}\right)^{\alpha+\frac{1}{2}}(t-s)^{-\alpha-\frac{1}{2}}+\left(\frac{t}{t-s}\right)^{\alpha+\frac{1}{2}}\left(\frac{t}{s}\right)^{\frac{1}{2}} s^{-\alpha}\right) \sup _{0 \leq r \leq s} \mathbb{E}\left\|Z_{r}^{\varepsilon}\right\|_{\alpha}^{2} d s \\
& \leq C+C t^{\alpha+\frac{1}{2}} \int_{0}^{t}\left((t-s)^{-\alpha-\frac{1}{2}} s^{-\alpha-\frac{1}{2}}\right) \sup _{0 \leq r \leq s} \mathbb{E}\left\|Z_{r}^{\varepsilon}\right\|_{\alpha}^{2} d s .
\end{aligned}
$$

As a consequence, by the Gronwall-type lemma (Lemma 7.6 in Nualart and Răşcanu [4]), we derive the desired estimate. Similarly, we can also show that $\sup _{t \in[0, T]} \mathbb{E}\left[\left\|X_{t}^{\varepsilon}\right\|_{\alpha}^{2}\right] \leq C$.

Lemma 3.2. Suppose that the averaged Eq. (3.2) satisfies the conditions (H1)-(H2). Then, for $0 \leq s<t \leq$ $T$, we have $\mathbb{E}\left[\left|Z_{t}^{\varepsilon}-Z_{s}^{\varepsilon}\right|^{2}\right] \leq C|t-s|$.

Proof: From (3.2), by conditions (H1-H2), we have

$$
\begin{aligned}
\mathbb{E}\left[\left|Z_{t}^{\varepsilon}-Z_{s}^{\varepsilon}\right|^{2}\right] & \leq 3 \varepsilon^{2} \mathbb{E}\left|\int_{s}^{t} \bar{f}\left(Z_{r}^{\varepsilon}\right) d r\right|^{2}+3 \varepsilon \mathbb{E}\left|\int_{s}^{t} \bar{\sigma}_{W}\left(Z_{r}^{\varepsilon}\right) d W_{r}\right|^{2}+3 \varepsilon^{2 H} \mathbb{E}\left|\int_{s}^{t} \bar{\sigma}_{H}\left(Z_{r}^{\varepsilon}\right) d B_{s}^{H}\right|^{2} \\
& \leq 3 \varepsilon_{0}^{2} \mathbb{E}\left|\int_{s}^{t} \bar{f}\left(Z_{r}^{\varepsilon}\right) d r\right|^{2}+3 \varepsilon_{0} \mathbb{E} \int_{s}^{t}\left|\bar{\sigma}_{W}\left(Z_{r}^{\varepsilon}\right)\right|^{2} d r+J_{3}(t, s) \\
& \leq C|t-s|^{2}+C|t-s|+J_{3}(t, s) .
\end{aligned}
$$

Then, by Lemma 3.1 and (2.1) and Proposition 4.1 in Nualart and Răşcanu [4], we have

$$
J_{3}(t, s):=3 \varepsilon_{0}^{2 H} \mathbb{E}\left|\int_{s}^{t} \bar{\sigma}_{H}\left(Z_{r}^{\varepsilon}\right) d B_{s}^{H}\right|^{2} \leq C_{\alpha, T} \Lambda^{2}\left(B^{H}\right)|t-s|^{2(1-\alpha)} \sup _{0 \leq r \leq T} \mathbb{E}\left\|Z_{r}^{\varepsilon}\right\|_{\alpha}^{2} \leq C|t-s|^{2(1-\alpha)} .
$$

Thus, we obtain the desired estimate. Similarly, we can also verify that $\mathbb{E}\left[\left|X_{t}^{\varepsilon}-X_{s}^{\varepsilon}\right|^{2}\right] \leq C|t-s|$.

Now, we claim the main theorem showing the relationship between solution processes $X_{t}^{\varepsilon}$ to the original Eq. (3.1) and $Z_{t}^{\varepsilon}$ to the averaged Eq. (3.2). It shows that the solution of averaged Eq. (3.2) converges to that of the original Eq. (3.1) in mean square sense and in convergence in probability, respectively.

Theorem 3.3. Suppose that orignal Eq. (3.1) and averaged Eq. (3.2) both satisfy the assumptions (H1)-(H2) and $(\mathrm{C} 1)-(\mathrm{C} 3)$. For a given arbitrarily small number $\delta_{1}>0$, there exist $L>0, \varepsilon_{1} \in\left(0, \varepsilon_{0}\right]$, such that for any $\varepsilon \in\left(0, \varepsilon_{1}\right]$, each $t \in\left[0, L \varepsilon^{-\gamma}\right], 0<\gamma \leq \min \left\{\frac{2 H-1}{2 \beta+2 H-1}, \frac{2 H-1}{2 H}\right\}<1$, we have $\sup _{t \in\left[0, L \varepsilon^{-\gamma}\right]} \mathbb{E}\left|X_{t}^{\varepsilon}-Z_{t}^{\varepsilon}\right|^{2} \leq \delta_{1}$.

Corollary 3.4. Suppose that all assumptions (H1)-(H2) and (C1)-(C3) are satisfied. Then for any number $\delta_{2}>0$, each $t \in\left[0, L \varepsilon^{-\gamma}\right]$, we have $\lim _{\varepsilon \rightarrow 0} \mathbb{P}\left[\sup _{t \in\left[0, L \varepsilon^{-\gamma}\right]}\left|X_{t}^{\varepsilon}-Z_{t}^{\varepsilon}\right|>\delta_{2}\right]=0$, where $L$ and $\gamma$ are the same to Theorem 3.3.

The Proof of Theorem 3.3: From (3.1) and (3.2), we have

$$
\begin{aligned}
\mathbb{E}\left|X_{t}^{\varepsilon}-Z_{t}^{\varepsilon}\right|^{2} \leq & 3 \varepsilon^{2} \mathbb{E}\left|\int_{0}^{t}\left(f\left(s, X_{s}^{\varepsilon}\right)-\bar{f}\left(Z_{s}^{\varepsilon}\right)\right) d s\right|^{2}+3 \varepsilon \mathbb{E}\left|\int_{0}^{t}\left(\sigma_{W}\left(s, X_{s}^{\varepsilon}\right)-\bar{\sigma}_{W}\left(Z_{s}^{\varepsilon}\right)\right) d W_{s}\right|^{2} \\
& +3 \varepsilon^{2 H} \mathbb{E}\left|\int_{0}^{t}\left(\sigma_{H}\left(s, X_{s}^{\varepsilon}\right)-\bar{\sigma}_{H}\left(Z_{s}^{\varepsilon}\right)\right) d B_{s}^{H}\right|^{2}=: 3 J_{1}(t)+3 J_{2}(t)+3 J_{3}(t),
\end{aligned}
$$

where $[0, t] \subset[0, u] \subset[0, T]$. So, for the first term, we have

$$
J_{1}(t)=\varepsilon^{2} \mathbb{E}\left|\int_{0}^{t}\left(f\left(s, X_{s}^{\varepsilon}\right)-\bar{f}\left(Z_{s}^{\varepsilon}\right)\right) d s\right|^{2} \leq C \varepsilon^{2} \mathbb{E}\left(\int_{0}^{t}\left|f\left(s, X_{s}^{\varepsilon}\right)-\bar{f}\left(Z_{s}^{\varepsilon}\right)\right| d s\right)^{2}
$$


and for the second term, we have

$$
J_{2}(t)=\varepsilon \mathbb{E}\left|\int_{0}^{t}\left(\sigma_{W}\left(s, X_{s}^{\varepsilon}\right)-\bar{\sigma}_{W}\left(Z_{s}^{\varepsilon}\right)\right) d W_{s}\right|^{2} \leq C \varepsilon \mathbb{E} \int_{0}^{t}\left|\sigma_{W}\left(s, X_{s}^{\varepsilon}\right)-\bar{\sigma}_{W}\left(Z_{s}^{\varepsilon}\right)\right|^{2} d s .
$$

For the last term, by Lemma 3.2, (2.1) and (H1), we have

where

$$
J_{3}(t) \leq \varepsilon^{2 H} \mathbb{E}\left|\int_{0}^{t}\left(\sigma_{H}\left(s, X_{s}^{\varepsilon}\right)-\bar{\sigma}_{W}\left(Z_{s}^{\varepsilon}\right)\right) s^{-\alpha} d s\right|^{2}+\varepsilon^{2 H} \mathbb{E}\left|\int_{0}^{t} \Sigma(s) d s\right|^{2},
$$

$$
\begin{aligned}
\Sigma(s)= & \int_{0}^{s} \mathbb{E}\left|\sigma_{H}\left(s, X_{s}^{\varepsilon}\right)-\bar{\sigma}_{H}\left(Z_{s}^{\varepsilon}\right)-\sigma_{H}\left(r, X_{r}^{\varepsilon}\right)+\bar{\sigma}_{H}\left(Z_{r}^{\varepsilon}\right)\right|(s-r)^{-1-\alpha} d r \\
& \leq \int_{0}^{s} \mathbb{E}\left|\sigma_{H}\left(s, X_{s}^{\varepsilon}\right)-\sigma_{H}\left(r, X_{r}^{\varepsilon}\right)\right|(s-r)^{-1-\alpha} d r+\int_{0}^{s} \mathbb{E}\left|\bar{\sigma}_{H}\left(Z_{s}^{\varepsilon}\right)-\bar{\sigma}_{H}\left(Z_{r}^{\varepsilon}\right)\right|(s-r)^{-1-\alpha} d r \\
& \leq C \int_{0}^{s}\left[(s-r)^{\beta}+(s-r)^{\frac{1}{2}}\right](s-r)^{-1-\alpha} d r \\
\leq & C s^{\beta-\alpha}+C s^{\frac{1}{2}-\alpha} .
\end{aligned}
$$

Thus, we have

$$
J_{3}(t) \leq \varepsilon^{2 H} u^{1-2 \alpha} \mathbb{E} \int_{0}^{t}\left|\sigma_{H}\left(s, X_{s}^{\varepsilon}\right)-\bar{\sigma}_{H}\left(Z_{s}^{\varepsilon}\right)\right|^{2} d s+C \varepsilon^{2 H} u^{2(1+\beta-\alpha)}+C \varepsilon^{2 H} u^{3-2 \alpha} .
$$

Finally, using conditions (H1-H2), we have

$$
\begin{aligned}
\mathbb{E}\left|X_{t}^{\varepsilon}-Z_{t}^{\varepsilon}\right|^{2} \leq & C \varepsilon^{2} \mathbb{E}\left(\int_{0}^{t}\left|f\left(s, X_{s}^{\varepsilon}\right)-\bar{f}\left(Z_{s}^{\varepsilon}\right)\right| d s\right)^{2}+C \varepsilon \mathbb{E} \int_{0}^{t}\left|\sigma_{W}\left(s, X_{s}^{\varepsilon}\right)-\bar{\sigma}_{W}\left(Z_{s}^{\varepsilon}\right)\right|^{2} d s \\
& +\varepsilon^{2 H} u^{1-2 \alpha} \mathbb{E} \int_{0}^{t}\left|\sigma_{H}\left(s, X_{s}^{\varepsilon}\right)-\bar{\sigma}_{H}\left(Z_{s}^{\varepsilon}\right)\right|^{2} d s+C \varepsilon^{2 H}\left(u^{2(1+\beta-\alpha)}+u^{(3-2 \alpha)}\right) \\
\leq & C\left(\varepsilon^{2} u+\varepsilon+\varepsilon^{2 H} u^{1-2 \alpha}\right) \mathbb{E} \int_{0}^{t}\left|X_{s}^{\varepsilon}-Z_{s}^{\varepsilon}\right|^{2} d s+C \varepsilon^{2 H}\left(u^{2(1+\beta-\alpha)}+u^{3-2 \alpha}\right) \\
& +C \varepsilon^{2} u^{2} J_{1}+C \varepsilon u J_{2}+\varepsilon^{2 H} u^{2-2 \alpha} J_{3} .
\end{aligned}
$$

According to conditions (C1-C3), Lemma 3.1 and the boundedness of $\varphi_{i}\left(T_{1}\right), T_{1} \in[0, T]$, we can obtain

Thus, we have

$$
\begin{aligned}
J_{1} & =\mathbb{E}\left(\frac{1}{t} \int_{0}^{t}\left|f\left(s, Z_{s}^{\varepsilon}\right)-\bar{f}\left(Z_{s}^{\varepsilon}\right)\right| d s\right)^{2} \leq C \sup _{0 \leq t \leq u}\left[\varphi_{1}^{2}(t)\right]\left(1+\sup _{0 \leq t \leq u} \mathbb{E}\left|Z_{t}^{\varepsilon}\right|^{2}\right), \\
J_{2} & =\mathbb{E} \frac{1}{t} \int_{0}^{t}\left|\sigma_{W}\left(s, Z_{s}^{\varepsilon}\right)-\bar{\sigma}_{W}\left(Z_{s}^{\varepsilon}\right)\right|^{2} d s \leq C \sup _{0 \leq t \leq u}\left[\varphi_{2}(t)\right]\left(1+\sup _{0 \leq t \leq u} \mathbb{E}\left|Z_{t}^{\varepsilon}\right|^{2}\right), \\
J_{3} & =\mathbb{E} \frac{1}{t} \int_{0}^{t}\left|\sigma_{H}\left(s, Z_{s}^{\varepsilon}\right)-\bar{\sigma}_{H}\left(Z_{s}^{\varepsilon}\right)\right|^{2} d s \leq C \sup _{0 \leq t \leq u}\left[\varphi_{3}(t)\right]\left(1+\sup _{0 \leq t \leq u} \mathbb{E}\left|Z_{t}^{\varepsilon}\right|^{2}\right) .
\end{aligned}
$$

$$
\begin{aligned}
\sup _{0 \leq t \leq u} \mathbb{E}\left|X_{t}^{\varepsilon}-Z_{t}^{\varepsilon}\right|^{2} \leq & C\left(\varepsilon^{2} u+\varepsilon+\varepsilon^{2 H} u^{1-2 \alpha}\right) \mathbb{E} \int_{0}^{u} \sup _{0 \leq r \leq s}\left|X_{r}^{\varepsilon}-Z_{r}^{\varepsilon}\right|^{2} d s \\
& +C \varepsilon^{2 H}\left(u^{2(1+\beta-\alpha)}+u^{3-2 \alpha}\right)+C\left(\varepsilon^{2} u+\varepsilon+\varepsilon^{2 H} u^{1-2 \alpha}\right) u .
\end{aligned}
$$

Then, for each $t \in\left[0, L \varepsilon^{-\gamma}\right]$, we have

$$
\sup _{0 \leq t \leq L \varepsilon^{-\gamma}} \mathbb{E}\left|X_{t}^{\varepsilon}-Z_{t}^{\varepsilon}\right|^{2} \leq Q \varepsilon^{1-\gamma}
$$

where $Q=C\left[\varepsilon^{2 H-1}\left(\varepsilon^{-\gamma(1+2 \beta-2 \alpha)}+\varepsilon^{-\gamma(2-2 \alpha)}+\varepsilon^{-\gamma(1-2 \alpha)}\right)+\varepsilon^{1-\gamma}+1\right] \times \exp \left(C\left(\varepsilon^{2-2 \gamma}+\varepsilon^{1-\gamma}+\varepsilon^{2 H-\gamma(2-2 \alpha)}\right)\right.$ is a constant if $\gamma \leq \min \left\{\frac{2 H-1}{2 \beta+2 H-1}, \frac{2 H-1}{2 H}\right\}<1$.

The Proof of Corollary 3.4: By the Chebyshev-Markov inequality and Theorem 3.3, for any given number $\delta_{2}>0$, let $\varepsilon \rightarrow 0$, one can find

$$
\mathbb{P}\left[\sup _{t \in\left[0, L \varepsilon^{-\gamma}\right]}\left|X_{t}^{\varepsilon}-Z_{t}^{\varepsilon}\right|>\delta_{2}\right] \leq \frac{1}{\delta_{2}^{2}} \sup _{t \in\left[0, L \varepsilon^{-\gamma}\right]} \mathbb{E}\left|X_{t}^{\varepsilon}-Z_{t}^{\varepsilon}\right|^{2} \leq \frac{Q}{\delta_{2}^{2}} \varepsilon^{1-\gamma}
$$

and the required result will be obtained.

Remark 3.5. If we consider SDEs driven by only a $\mathrm{fBm}\left(\sigma_{W}=0\right)$, the equation (1.1) can be rewrited as a deterministic differential equation, then, use the pathwise approach, for a given arbitrarily small number $\delta_{3}>$ 0 , there exist $L>0, \varepsilon_{1} \in\left(0, \varepsilon_{0}\right]$, such that for any $\varepsilon \in\left(0, \varepsilon_{1}\right]$, each $t \in\left[0, L \varepsilon^{-\gamma}\right], \gamma \leq \min \left\{\frac{H}{4 H-1}, \frac{H}{2 \beta+2 H-1}\right\}$, we have, $\sup _{t \in\left[0, L \varepsilon^{-\gamma}\right]}\left|X_{t}^{\varepsilon}-Z_{t}^{\varepsilon}\right| \leq \delta_{3}, \delta_{3}=Q \varepsilon^{\frac{H-\gamma}{2}}, Q$ is a constant. Here, we omit the proof. 
Remark 3.6. Moreover, in this paper, instead of $\mathrm{fBm}$ one can take any process, which is almost surely Hölder continuous with Hölder exponent greater than $\frac{1}{2}$. The results will be effective.

\section{Aknowledgement}

The research of B. Pei was partially supported by the NSF of China (11802216), the China Postdoctoral Science Foundation (2019M651334) and JSPS KAKENHI Grant Number JP18F18314. The research of Y. Xu was partially supported by the NSF of China (11702216), Shaanxi Province Project for Distinguished Young Scholars and the Fundamental Research Funds for the Central Universities. The authors are very grateful to Prof. Yuzuru Inahama for his encouragement and useful discussions.

\section{References}

[1] A. Kolmogorov, Wienersche Spiralen und einige andere interessante Kurven im Hilbertschen, Raum, $C$. R. (Dokaldy) Acad. Sci. URSS (N.S.), 26 (1940), 115-118.

[2] B. Mandelbrot and J. Van Ness, Fractional Brownian motions, fractional noises and applications, SIAM Review, $10: 422-427,1968$.

[3] M. Zähle. Integration with respect to fractal functions and stochastic calculus. I. Probability Theory and Related Fields, 111(3): 333-374,1998.

[4] D. Nualart and A. Răşcanu. Differential equations driven by fractional Brownian motion. Collectanea Mathematica, 53(1): 55-81,2002.

[5] J. Guerra, D. Nualart. Stochastic differential equations driven by fractional Brownian motion and standard Brownian motion. Stochastic Analysis and Applications, 26(5): 1053-1075,2008.

[6] T. Lyons. Differential equations driven by rough signals (I): An extension of an inequality of LC Young. Mathematical Research Letters, 1(4): 451-464,1994.

[7] K. Kubilius. The existence and uniqueness of the solution of an integral equation driven by a psemimartingale of special type. Stochastic Processes and Their Applications, 98(2): 289-315,2002.

[8] Y. Xu, R. Guo, D. Liu, H. Zhang and J. Duan. Stochastic averaging principle for dynamical systems with fractional Brownian motion. Discrete 8 Continuous Dynamical Systems-Series B, 19(4):1197-1212, 2014.

[9] L. Young. An inequality of Hölder type, connected with Stieljes integration. Acta Mathematica, 67:251$282,1936$.

[10] L. Decreusefond and A. Üstünel. Fractional Brownian motion: Theory and applications. ESAIM: Proceedings, 5:75-86, 1998.

[11] R. Khasminskii. A limit theorem for the solutions of differential equations with random right-hand sides. Theory of Probability \& Its Applications, 11(3):390-406, 1966.

[12] W. Mao, L. Hu, S. You and X. Mao. The averaging method for multivalued SDEs with jumps and non-Lipschitz coefficients. Discrete $\&$ Continuous Dynamical Systems-B, 19:19-36,2019.

[13] J. Xu, J. Liu. An averaging principle for Multivalued stochastic differential equations. Stochastic Analysis and Applications, 32:962-974,2014.

[14] Y. Xu, J. Duan, and W. Xu. An averaging principle for stochastic dynamical systems with Lévy noise. Physica D: Nonlinear Phenomena, 240(17):1395-1401, 2011.

[15] Y. Xu, B. Pei, and J.-L. Wu. Stochastic averaging principle for differential equations with non-Lipschitz coefficients driven by fractional Brownian motion. Stochastics $\&$ Dynamics, 17(02): 1750013,2017. 
[16] Y. Xu, B. Pei, and R. Guo. Stochastic averaging for slow-fast dynamical systems with fractional Brownian motion. Discrete \& Continuous Dynamical Systems-Series B, 20(7):2257-2267, 2015.

[17] R. Guo and B. Pei. Stochastic averaging principles for multi-valued stochastic differential equations driven by Poisson point processes. Stochastic Analysis and Applications, 36(4) : 751-766, 2018. 